\title{
Effect of diet on the fatty acid composition of the major phospholipids of infant cerebral cortex
}

James Farquharson, E Cherry Jamieson, Kurshid A Abbasi, W J Ainslie Patrick, Robert W Logan, Forrester Cockburn

\begin{abstract}
The fatty acid compositions of the major cerebral cortex phospholipids, phosphatidylcholine, phosphatidylethanolamine, and phosphatidylserine were measured in 16 term and one preterm 'cot death' infants fed exclusively either breast milk or one of two formulas. Docosahexaenoic acid (DHA; C22:6n-3) content in cerebral cortex phosphatidylethanolamine and phosphatidylserine of breast fed infants was greater than in both formula groups with significances varying between $p<0.01$ and $p<0.001$. Compensation for this deficiency in DHA in the formula fed infants was largely achieved by increased incorporation of docosapentaenoic acid (C22:5n-6) in the cerebral cortex of term infants and Mead (C20:3n-9) and dihomo Mead acids $(C 22: 3 n-9)$ in the preterm infant.

As the phospholipids most affected are known to perform an important role in membrane function and are possibly integral to neurotransmission it is recommended that breast milk substitute infant formulas should contain $n-3$ and $n-6$ series polyunsaturated fatty acids in proportions similar to those of human milk.
\end{abstract}

(Arch Dis Child 1995; 72: 198-203)

Keywords: infant, brain, phospholipids, milks.

At term the newborn human infant brain weighs about $350 \mathrm{~g}$, which is approximately $10 \%$ of the total body weight. During the first year brain weight increases by about $750 \mathrm{~g}$ so that the brain remains approximately $10 \%$ of the body weight. This threefold increase of weight from birth is achieved largely by nerve cell growth with concurrent dendritic arborisation, glial cell (astrocyte and oligodendrocyte) proliferation, and axon myelination. Maximal glial cell proliferation in term infants takes place in the first six postnatal months. ${ }^{1}$ The cerebral cortex (grey matter) is composed largely of neurones and astrocytes and makes up about $45 \%$ of the total brain weight. Much of the increase in the grey matter weight is due to the development of the complex arborisations and synaptosome formation which subserve neuronal function and the learning processes. Myelination also proceeds rapidly after birth and in this process neuroglial cells envelop the axons of cortical neurones with sheaths of myelin which speed the rate of transmission of electrical messages between neurones, other central nervous system cells, and end organs such as muscle and skin.

Approximately $60 \%$ of the total energy intake of the infant during the first year is utilised by the brain and much of this energy is used to synthesise neuronal membrane and deposit myelin. Fatty acids from human milk or infant formulas provide not only a source of hydrocarbon for energy production but help synthesise the complex hydrocarbon structures necessary for the creation of neurotransmitter membranes.

Breast fed infants have significantly greater concentrations of the long chain polyunsaturated fatty acid (PUFA), docosahexaenoic acid (DHA; C22:6n-3) in their cerebral cortex phospholipids than infants fed current infant formulas. ${ }^{2}$ Cerebral cortex neuronal membrane phospholipids are composed of phosphatidylcholine, phophatidylethanolamine, phosphatidylserine, and phosphatidylinositol. While the membrane receptor and secondary messenger characteristics of phosphatidylinositol and its metabolites have been extensively investigated ${ }^{4-6}$ the functions of other phospholipids are less certain. Phosphatidylcholine is known to confer a stabilising influence within the neuronal membrane. ${ }^{7}$ The carboxyl groups of phosphatidylserine function as ion exchange sites $^{8}$ while both phosphatidylserine and phosphatidylethanolamine have an important influence on the distribution of protein molecules in the membrane. ${ }^{79}$ Incorporation of proteins into the phosphatidylserine and phosphatidylethanolamine rich areas of membrane is critically dependent on the chain length, degree of unsaturation, and hence configuration of the two fatty acids attached to each phosphatidylethanolamine moiety. ${ }^{10}$ The major DHA containing phospholipids of synaptosomes are phosphatidylserine and phosphatidylethanolamine and the polyunsaturated DHA of these moieties will preferentially cross link to proteins. The degree of unsaturation present in neuronal phospholipid fatty acids can mediate the activities of 
Table 1 Infant details and milk essential fatty acid compositions in relation to diet

\begin{tabular}{llcc}
\hline & Diet & & \\
\cline { 2 - 4 } & Breast milk & $S M A$ & CGOST \\
\hline Infant data & & & \\
Mean (SD) birth weight (g) & $3235(574)$ & $3011(593)$ & $3044(475)$ \\
Mean (SD) gestational age (weeks) & $39 \cdot 8(1 \cdot 5)$ & $39 \cdot 2(1 \cdot 3)$ & $39 \cdot 5(1 \cdot 7)$ \\
Mean (range) age (weeks) & $15 \cdot 8(6-38)$ & $20 \cdot 5(6-43)$ & $21 \cdot 8(9-40)$ \\
Range Apgar score & $7-9$ & $8-9$ & $8-9$ \\
$\quad 1$ min & $9-10$ & 9 & $9-10$ \\
$\quad 5$ min & $2 / 3$ & $6 / 0$ & $3 / 2$ \\
Male/female & $11 \cdot 9(6 \cdot 3-15 \cdot 7)$ & $16 \cdot 0$ & $14 \cdot 5$ \\
Milk essential fatty acids (wt $\%)^{\star}$ & $0 \cdot 8(0 \cdot 5-1 \cdot 2)$ & $1 \cdot 5$ & $0 \cdot 4$ \\
Linoleic (C18:2n-6) & $0 \cdot 5(0 \cdot 3-0 \cdot 8)$ & ND & ND \\
$\alpha$-Linolenic (C18:3n-3) & $0 \cdot 4(0 \cdot 1-0 \cdot 5)$ & ND & ND \\
Arachidonic (C20:4n-6) & & \\
Docosahexaenoic (C22:6n-3) & & & \\
\hline
\end{tabular}

*Results given as median (range) for 13 mature human breast milks; results for SMA and CGOST are mean values provided by milk manufacturers. ND $=$ not detected or $<0.05 \%$ of total fatty acids.
LIPID EXTRACTION AND FATTY ACID ANALYSES Phospholipids were isolated from the parietal cortex as described. ${ }^{2}$ These were separated by two dimensional silica gel thin layer chromatography (Silica Gel G, Merck) initially in chloroform:methanol: water $(65: 25: 4 \mathrm{v} / \mathrm{v} / \mathrm{v})$ followed by development in $\mathrm{n}$-butanol:acetic acid:water $(60: 20: 20 \mathrm{v} / \mathrm{v} / \mathrm{v})$. On a second plate the phospholipid standards $(10 \mathrm{mg} / \mathrm{ml}$ in chloroform:methanol, 95:5 $\mathrm{v} / \mathrm{v}), \quad \mathrm{L}-\alpha$-phosphatidylcholine, dipalmitoyl; L- $\alpha$-phosphatidylethanolamine, dipalmitoyl; L- $\alpha$-phosphatidyl-L-serine (bovine brain); L- $\alpha$-phosphatidylinositol; sphingomyelin (bovine brain); tripalmitin; cholesteryl stearate (all purchased from Sigma Chemical Co) were separated by the same procedure. After drying, the plates, sprayed with $2^{\prime}, 7^{\prime}$-dichlorofluorescein (1

\section{DATA AND STATISTICAL ANALYSIS}

The composition of each cerebral cortex phospholipid FAME was expressed as a weight percentage of the total fatty acids identified. For each phospholipid only the eight most abundant fatty acids were considered (the results being rounded to $100 \%$ ), leading to the exclusion of fatty acids contributing $<0.6 \%$ to the total. The percentage content of essential fatty acids and their metabolites, arachidonic acid and DHA in breast milk were given without exclusion of fatty acids contributing $<0.6 \%$ to the total.

All group results are presented for each phospholipid as means and standard deviations with statistical significance calculated by the two tailed, Student's $t$ test with eight or nine degrees of freedom. It was apparent that the distribution of the CGOST docosapentaenoic acid (DPA; C22:5n-6) results in phosphatidylserine was skewed and these data

Table 2 Fatty acid profile in cerebral cortex phosphatidylcholine related to infant diet

\begin{tabular}{lrrr}
\hline & \multicolumn{1}{l}{ Diet } \\
\cline { 2 - 4 } Fatty acid & \multicolumn{1}{c}{ Breast milk } & \multicolumn{1}{c}{ SMA } & \multicolumn{1}{c}{ CGOST } \\
\hline C14:0 & $1 \cdot 9(0 \cdot 4)$ & $2 \cdot 0(0 \cdot 5)$ & $1 \cdot 8(0 \cdot 7)$ \\
C16:0 & $53 \cdot 8(1 \cdot 8)$ & $54 \cdot 4(1 \cdot 8)$ & $53 \cdot 3(2 \cdot 8)$ \\
C16:1n-7 & $4 \cdot 3(1 \cdot 3)$ & $4 \cdot 4(0 \cdot 4)$ & $4 \cdot 4(1 \cdot 3)$ \\
C18:0 & $9 \cdot 4(1 \cdot 0)$ & $9 \cdot 7(1 \cdot 2)$ & $9 \cdot 9(1 \cdot 6)$ \\
C18:1n-9 & $25 \cdot 8(0 \cdot 7)$ & $24 \cdot 5(1 \cdot 3)$ & $24 \cdot 4(1 \cdot 8)$ \\
C18:2n-6 & $0 \cdot 7(0 \cdot 2)$ & $0 \cdot 9(0 \cdot 1)$ & $1 \cdot 0(0 \cdot 2)$ \\
C20:3n-6 & $0 \cdot 9(0 \cdot 2)$ & $1 \cdot 0(0 \cdot 2)$ & $1 \cdot 1(0 \cdot 1)$ \\
C20:4n-6 & $3 \cdot 2(0 \cdot 7)$ & $3.3(1 \cdot 4)$ & $3.9(0 \cdot 6)$ \\
Total (n-6) & $4 \cdot 7(0 \cdot 8)$ & $5 \cdot 1(1 \cdot 7)$ & $5 \cdot 9(0 \cdot 8)$ \\
& & & \\
\hline
\end{tabular}

^Mean (SD) weight percentage of total fatty acids present.

\begin{abstract}
$\mathrm{mg} / \mathrm{ml}$ methanol), were viewed under ultraviolet light and the lipids in the specimen extracts identified by comparison with the standards. The areas of silica corresponding to the phospholipids phosphatidylcholine, phosphatidylethanolamine, and phosphatidylserine were scraped off the plate and added directly to the derivatisation reagent $(2 \mathrm{ml}$, boron trifluoride:methanol, $14 \% \mathrm{w} / \mathrm{v})$. The fatty acid methyl esters (FAME) formed were separated by gas chromatography. ${ }^{2}$ Identification of the fatty acids extracted from the human milk samples was achieved using a method previously employed in analysing subcutaneous fat triglycerides. ${ }^{22}$

membrane bound enzymes.
an analysis of cerebral cortex phosphatidylcholine, phophatidylethanolamine, and phosmilks in the first months of life and consider how the biochemical findings might relate to
\end{abstract}

\begin{abstract}
composition unique to the catchment area of the study we subsequently analysed 13 random mature human milk samples and compared the

results with published data. ${ }^{20} 21$ Cow and Gate Premium (Cow and Gate) formulas. The Cow and Gate and Osterfeed were combined as a designat fatty acid composition of both milks at the population involved precluded specific matching of individuals in each feeding group. related variations in cerebral cortex phosphofatty acids of term infants, in breast fed (two months) before receiving nonermula milks. ${ }^{19}$ However, we sought groups, together with near equivalence in age ranges. Cortical tissue from a 10 week old, 30 week gestation infant fed SMA was also examined. To ensure that the breast fed infants
\end{abstract}


Table 3 Fatty acid profile in cerebral cortex

phosphatidylethanolamine related to infant diet

\begin{tabular}{|c|c|c|c|}
\hline \multirow[b]{2}{*}{ Fatty acid ${ }^{\star}$} & \multicolumn{3}{|l|}{ Diet } \\
\hline & Breast milk & $S M A$ & CGOST \\
\hline $\begin{array}{l}\text { C16:0 } \\
\text { C18:0 } \\
\text { C18:1n-9 } \\
\text { C20:3n-6 } \\
\text { C20:4n-6 } \\
\text { C22:4n-6 } \\
\text { C22:5n-6 } \\
\text { C22:6n-3 } \\
\text { Total (n-6) } \\
\text { Total (n-6+n-3) }\end{array}$ & $\begin{array}{r}8 \cdot 4(2 \cdot 1) \\
30 \cdot 8(2 \cdot 6) \\
8 \cdot 8(1 \cdot 5) \\
1.5(0 \cdot 1) \\
17 \cdot 6(0 \cdot 7) \\
12 \cdot 0(0 \cdot 8) \\
3 \cdot 2(0 \cdot 6) \\
17 \cdot 7(1 \cdot 3) \\
34 \cdot 3(1 \cdot 7) \\
52 \cdot 0(2 \cdot 1)\end{array}$ & $\begin{array}{c}6.4(0.9) \\
31.4(1.6) \\
9.2(0.8) \\
2.0(0.4) \rrbracket \\
20.1(0.8) \ddagger \\
12.6(1.5) \\
4.8(0.7) \oint \\
13.4(1.2) \ddagger \\
39.6(2.3) \oint \\
53.0(2 \cdot 1)\end{array}$ & 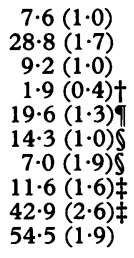 \\
\hline
\end{tabular}

${ }^{\star}$ Mean (SD) weight percentage of total fatty acids present. $\mathrm{tp}<0.05 ; \neq \mathrm{p}<0.001 ; \S \mathrm{p}<0.01 ;$ qp $<0.02$ all calculated by Student's $t$ test.

were therefore also analysed by the nonparametric Mann-Whitney U test. A similar tendency was noted in the breast milk results which are given as median and ranges.

\section{Results}

The infant characteristics and milk essential fatty acid compositions are illustrated in table 1. The essential fatty acid content of human milk from our local population of mothers was very similar to those reported by other workers. $^{2021}$ No significant differences were found between feeding groups in the major cerebral cortical phosphatidylcholine fatty acids (table 2). Analysis of the phosphatidylethanolamine and phosphatidylserine fractions also revealed no significant differences between groups in the content of saturated and monounsaturated fatty acids (tables 3 and 4). DHA was greater in the phosphatidylethanolamine of the breast fed group than both formula fed groups $(p<0.001)$ and the distribution of results can be seen in fig 1 . Formula fed infants 'compensated' by increased incorporation of all the major n-6 series PUFAs (table 3), such that in all feeding groups PUFAs accounted for over $50 \%$ of total phosphatidylethanolamine fatty acids. In phosphatidylethanolamine the only significant difference between the formula fed infants was a greater DPA in the CGOST group $(p<0.05)$ as shown in fig 2 . The phosphatidylserine fraction represents about $15 \%$ of total cerebral cortex phospholipids in the first year of life and the breast fed group has a greater DHA con-

Table 4 Fatty acid profile in cerebral cortex phosphatidylserine related to infant diet

\begin{tabular}{|c|c|c|c|}
\hline \multirow[b]{2}{*}{ Fatty acid ${ }^{\star}$} & \multicolumn{3}{|l|}{ Diet } \\
\hline & Breast milk & $S M A$ & CGOST \\
\hline $\begin{array}{l}\text { C16:0 } \\
\text { C18:0 } \\
\text { C18:1n-9 } \\
\text { C20:3n-6 } \\
\text { C20:4n-6 } \\
\text { C22:4n-6 } \\
\text { C22:5n-6 } \\
\text { C22:6n-3 } \\
\text { Total (n-6) } \\
\text { Total }(n-6+n-3)\end{array}$ & $\begin{array}{r}2.7(1 \cdot 1) \\
44.6(1 \cdot 7) \\
6.3(2 \cdot 6) \\
1.7(0.3) \\
7.9(1 \cdot 6) \\
7.9(1 \cdot 1) \\
5.3(1 \cdot 5) \\
23.5(1.3) \\
22 \cdot 8(2.9) \\
46.4(2 \cdot 8)\end{array}$ & $\begin{array}{c}2 \cdot 2(0.9) \\
44 \cdot 6(1 \cdot 5) \\
6 \cdot 8(0.8) \\
2 \cdot 0(0.4) \\
9 \cdot 0(1.4) \\
8 \cdot 5(0.9) \\
7.7(0.9) \\
19 \cdot 3(1.8) \\
27 \cdot 1(1.9) 9 \\
46.5(0.9)\end{array}$ & $\begin{array}{c}2.8(1 \cdot 3) \\
43.7(0.9) \\
8.9(2 \cdot 0) \\
2.3(0.5) \dagger \\
9.0(1 \cdot 1) \\
9 \cdot 3(1 \cdot 2) \\
10.4(3.1) 9 \\
14 \cdot 4(1.5) \ddagger \\
31.0(3.5) \oint \\
45.4(3.1)\end{array}$ \\
\hline
\end{tabular}

* Mean (SD) weight percentage of total fatty acids present. $+\mathrm{p}<0.05 ; \neq \mathrm{p}<0.001 ; \oint \mathrm{p}<0.01 ;$ qp $<0.02$ all calculated by Student's $t$ test.

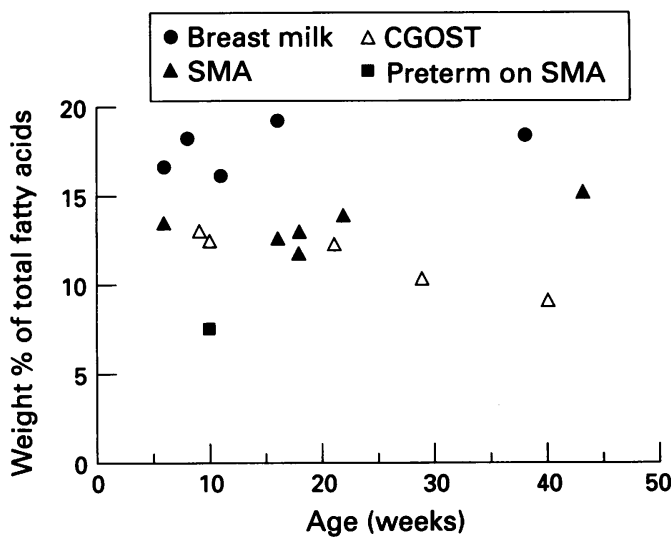

Figure 1 Distribution of cerebral cortex phosphatidylethanolamine DHA (C22:6n-3) in relation to infants' diet and age.

tent than both the SMA $(p<0.01)$ and CGOST $(p<0.001)$ groups (table 4$)$. The individual, age related DHA concentrations are shown in fig 3. A 'compensatory' effect was again evident with increased insertion of DPA into the phosphatidylserine fraction of both formula fed groups (fig 4), such that total PUFA content was similar in each group (table 4). The one significant difference between the formula fed infants was a greater DHA in the SMA group than in the CGOST group $(p<0.001)$. The lowest DHA content encountered in the phosphatidylethanolamine fraction $(7 \cdot 6 \%)$ was from the 10 week old preterm, SMA fed infant (fig 1). In the phosphatidylserine the DHA at $12.9 \%$ (fig 3) was as for the phosphatidylethanolamine about $6 \%$ lower than that found in the term SMA fed individuals. In this preterm infant overall total PUFA concentrations were, however, maintained at about $50 \%$ in each phospholipid by the incorporation of $\mathrm{n}-9$ series Mead acid (C20:3) and dihomo Mead acid (C22:3) in the phosphatidylserine $(1 \cdot 1 \%$ and $1.6 \%)$ and phosphatidylethanolamine fractions $(2.5 \%$ and $1.7 \%$ ) respectively. Application of nonparametric, two tailed Mann-Whitney $U$ test statistics to the phosphatidylserine DPA concentrations confirmed that both the formula fed groups, SMA $(p<0.05)$ and CGOST $(p<0 \cdot 02)$, contained higher percentage contents than the breast fed group.

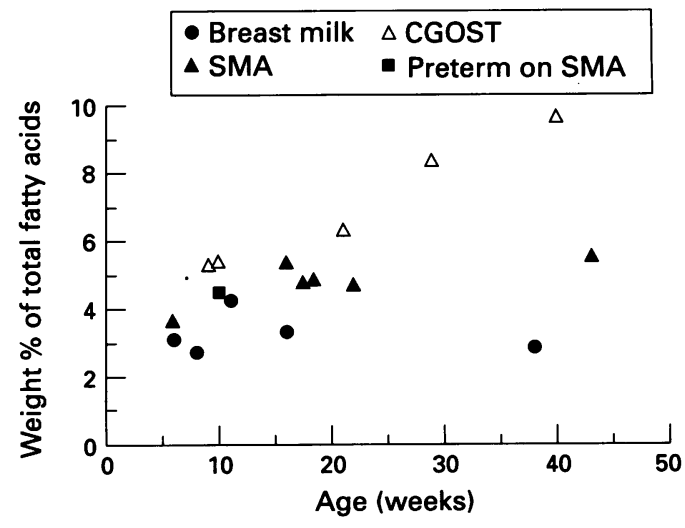

Figure 2 Distribution of cerebral cortex phosphatidylethanolamine DPA (C22:5n-6) in relation to infants' diet and age. 


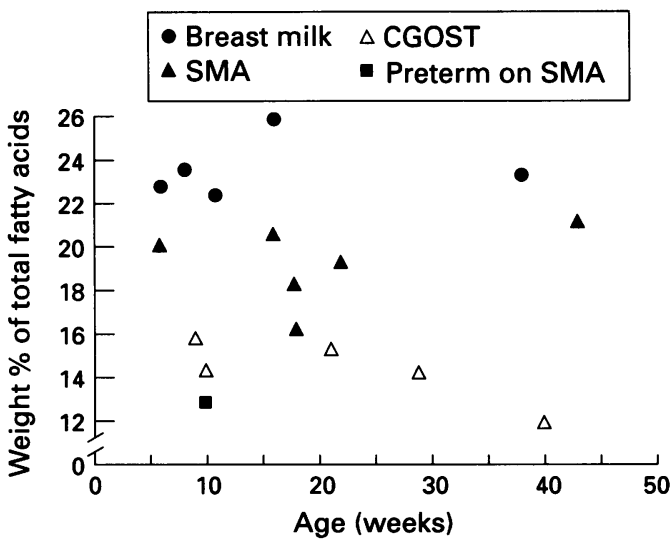

Figure 3 Distribution of cerebral cortex phosphatidylserine $D H A(C 22: 6 n-3)$ in relation to infants' diet and age.

\section{Discussion}

All membranes whether they are cell surface or form part of an intracellular organelle such as mitochondrion or peroxisome are composed of phospholipid bilayers. Phospholipids are amphilic (like both water and lipid) because they have chemical groupings at one end which are hydrophilic (like water) and at the other end hydrophobic (dislike water). ${ }^{1}$ This property allows these molecules to permit interaction between a wide range of water soluble and fat soluble substances while limiting movement of water and other substances between the outside and inside of the membrane. Membrane lipid provides a flexible and adaptable structure into which are inserted proteins and glycoproteins such as enzymes, transmembrane transporter proteins or receptors. The main membrane phospholipids contain two fatty acids and a substituted (amino) alcohol attached to a glycerol phosphate backbone which is hydrophilic. The nature of the alcohol head group and the attached fatty acids have major effects on the biophysical function of that membrane. ${ }^{23}$ Saturated fatty acids and those containing trans double bonds tend to adopt a straighter and more rigid configuration. Ethylenic double bonds in monounsaturated and polyunsaturated fatty acids provide a site of chemical reactivity. Thus membranes with phospholipids containing higher concentrations of $c i s$ double

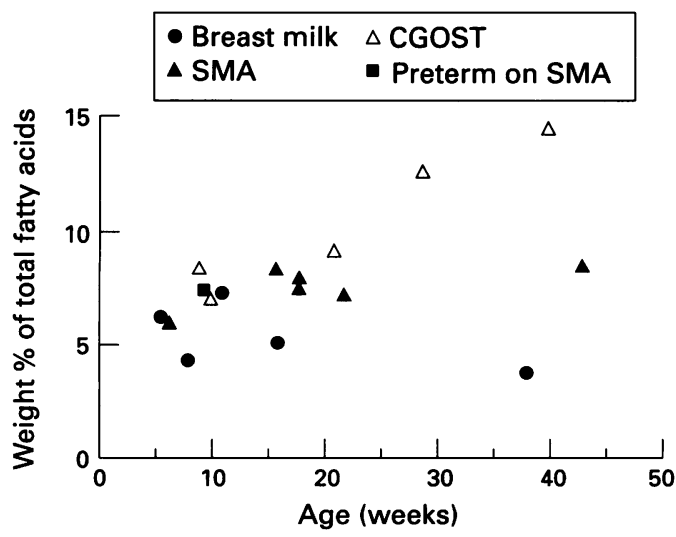

Figure 4 Distribution of cerebral cortex phosphatidylserine DPA (C22:5n-6) in relation to infants' diet and age. bonds are more flexible and more permeable. It is the presence of high concentrations of DHA on phosphatidylethanolamine and phosphatidylserine, particularly at the inner aspect of the phospholipid bilayer that allows rapid and repeated complex biochemical activities to take place at the neuronal synaptosomes. ${ }^{102324}$ DHA has a highly specific distribution and is the predominant membrane fatty acid of synaptosomes, retinal photoreceptors, mitochondria, and spermatozoa. Membrane thickness, elasticity, porosity, and ability to support or transmit other molecules also depends on the organic bases such as choline, ethanolamine, serine, and inositol which are attached to the phosphoglycerides. ${ }^{23}$ The control mechanism that determines the siting and type of phospholipid in the lipid bilayers of membranes is unknown. Whereas protein synthesis will cease if essential amino acid supplies are deficient, incorporation of fatty acids into membrane phospholipids will proceed and it appears from the data presented that the next nearest available fatty acid is substituted thus altering the properties of that membrane. Stability of mammalian membranes is crucially dependent upon the presence of long chain PUFAs. ${ }^{25}$ In severe fatty acid deficiency states PUFAs of the n-3 and n- 6 series are replaced by PUFAs of the n-9 family. ${ }^{26}$ The membrane becomes metabolically unstable and more permeable to water. Where there is a lesser deficiency of $n-3$ series, then $n-6$ fatty acids alone may substitute for them.

The reductions in the DHA content of phosphatidylserine and phosphatidylethanolamine between the breast and artificially fed infants are of a magnitude which, in vitro, would be sufficient to alter membrane function and could therefore critically affect the resultant responses to electrical and chemical stimulation and alter membrane and neurotransmitter function. It can be seen from figs 2 and 4 that there is a preferential replacement of DHA in the phospholipid membrane by DPA. This selective substitution, although reported in animal studies, ${ }^{27-29}$ has not hitherto been identified in man. It appears that it is only from about the fourth month of life (figs 2 and 4) that DHA is specifically replaced by DPA and this is particularly prominent in the CGOST group. Synthesis of both DHA and DPA from the parent essential fatty acids, $\alpha$-linolenic and linoleic respectively, are probably ultimately dependent on a peroxisomal $\beta$-oxidation reaction (' $\Delta 4$-desaturase'), ${ }^{30}$ which may not function in early infancy. This may be the major reason why DHA is initially replaced by less unsaturated $n-6$ series PUFAs (tables 3 and 4).

It appears that the DHA content of phosphatidylserine in cortical neuronal membranes is 'safeguarded' and preferentially incorporated when compared with the degree of substitution of DHA with DPA seen in phosphatidylethanolamine. The subcutaneous fat reserves of the parent essential fatty acid $\alpha$-linolenic acid are negligible at birth and those reserves of DHA which are present are rapidly exhausted. ${ }^{22}$ From the data presented 
in tables 3 and 4 it can be seen that the DHA concentrations in the phosphatidylserine of the SMA group are nearer to those of the breast fed infants, although still significantly lower (table 4). This increased DHA must have been synthesised from dietary $\alpha$-linolenic acid and preferentially distributed to phosphatidylserine as no significant differences in DHA were found between the formula fed groups in the phosphatidylethanolamine fraction. From fig 3 it can be seen that there is one breast fed infant with the highest DHA content $(25 \cdot 8 \%)$. This infant had a very high concentration of $\alpha$-linolenic acid $(0.9 \%)$ in subcutaneous triglyceride. ${ }^{22}$ It appears that optimal synthesis of DHA does not occur until an $\alpha$-linolenic substrate concentration in excess of $1 \%$ of total fatty acids is present in the diet, although this value was found in only $30 \%$ of breast milks analysed. With human milk, however, DHA is provided directly from the milk feed so that the need for synthesis of DHA from $\alpha$-linolenic acid is not so critical in the breast fed infant. Preformed long chain PUFAs are incorporated into the developing rat brain with a greater than tenfold efficiency when compared with those synthesised from the parent essential fatty acids. ${ }^{31}$ The brain preferentially uses the preformed long chain fatty acids and not those synthesised from the parent essential fatty acids. It is important to note that there are major differences in the DHA content of phosphatidylethanolamine and phosphatidylserine between breast and artificially fed infants even at 40 weeks after birth in term infants.

The finding of significant quantities of Mead acid and dihomo Mead acid in the phosphatidylserine and phosphatidylethanolamine fractions of the infant born preterm is very worrying. These fatty acids are very unstable and are most unlikely to allow the membranes to function normally.

There is clustering of phosphatidylserine, phosphatidylethanolamine, and phosphatidylcholine head groups into membrane areas known as domains. The phosphatidylserine and phosphatidylethanolamine domains are critically important for neurotransmitter function. It has long been recognised that there are structural similarities between phosphoserine, which is the hydrophilic moiety of phosphatidylserine, and the major central nervous system activatory neurotransmitter glutamate (or aspartate) and the polar phosphoethanolamine of phosphatidylethanolamine, which resembles the inhibitory amino acid neurotransmitter gamma aminobutyric acid (GABA). The phospholipids phosphatidylethanolamine and phosphatidylserine are known to exchange polar head groups directly in vivo and may also be interconverted in a simple irreversible decarboxylation reaction similar to that which neutralises the effect of glutamate by its conversion to GABA. ${ }^{32}$ Although we have no direct evidence that phosphatidylserine functions in tandem with glutamate it is known that L-AP4 (L-2-amino-4-phosphonobutanoic acid), a phosphorus containing molecule structurally similar to phosphoserine is a proved L-glutamate agonist at presynaptic terminals and retinal bipolar receptors. ${ }^{33-35}$ It may be that phosphatidylserine and phosphatidylethanolamine have a neurotransmitter function that could be independent or work in parallel with the glutamate-GABA system.

We have, therefore, demonstrated major differences in the fatty acid composition of phosphatidylethanolamine and phosphatidylserine in the brains of artificially fed infants compared with those who have been breast fed. What if anything is the clinical consequence of these findings? Lucas and his colleagues demonstrated that preterm infants fed human milk have a higher developmental status at 18 months and higher intelligence quotient in later childhood than those fed infant formulas. ${ }^{36} 37$ Preterm infants fed standard formulas have significantly different electroretinographic patterns indicating a delayed rod photoreceptor maturation compared with those fed human milk or given supplementary DHA. Visual cortical functions as measured by pattern reversal, visually evoked potential, and forced choice preferential looking visual acuity response are also better in breast fed or fish oil supplemented preterm infants. ${ }^{38}$ In a study of experimental infant formulas providing $0 \cdot 2 \%$ DHA, Carlson et al have shown that DHA supplemented preterm infants have greater red blood cell phosphatidylethanolamine DHA content and better visual acuity than standard formula fed preterm infants. ${ }^{39} \mathrm{DHA}$ supplemented infants performed significantly better than controls on the Bayley mental scales. It has also been shown that term infants' visual responses at five months after birth correlate with erythrocyte DHA concentrations ${ }^{40}$ and that human milk fed infants have better stereoacuity and better matching ability at 3 years of age than do formula fed children. ${ }^{41}$ The British Paediatric Association Standing Committee on Nutrition has recently reviewed the differences in cognitive function between breast and bottle fed infants. ${ }^{42}$ In spite of the difficulties of interpretation of many confounding variables in the published evidence their conclusion was that there is a factor in breast milk that if fed for four or more months is likely to be responsible for the greater cognitive function in breast fed infants. They further suggest that DHA might be that factor. The evidence we present suggests possible mechanisms for altered neurotransmission in DHA deficient brains of formula fed infants. Infants have no reserve pool of DHA and there is limited ability to synthesise DHA from the essential fatty acid precursor $\alpha$-linolenic acid, therefore DHA should be considered an essential fatty acid for at least the first four months of life in term infants and possibly longer in preterm infants. There must now be little doubt that there is a 'short term' effect of DHA deficiency on efficiency of synaptic transmission during a critical period of brain development and learning. There is so much 'redundancy' and 'reserve' in the efficiency of central nervous function that the biochemical differences demonstrated in the brains of these infants appears to convey only a 
'marginal' handicap on artificially fed infants. However, these short term effects and indeed the long term effects on neuronal integrity that might predispose to adult neurodegenerative disease ${ }^{43-46}$ warrant urgent attention.

The authors are indebted to Mrs Myra Fergusson and Miss Jean Hyslop for their assistance in the preparation of this manuscript.

1 Dobbing J. The later development of the brain and its vulnerability. In: Davis JA, Dobbing J, eds. Scientific foundations of paediatrics. 2nd Ed. London: William Heineman, 1981: 744-59.

2 Farquharson J, Cockburn F, Patrick WA, Jamieson EC, Logan RW. Infant cerebral cortex phospholipid fatty acid composition and diet. Lancet 1992; 340: 810-3.

3 Makrides M, Neumann MA, Byard RW, Simmer K, Gibson RA. Fatty acid composition of brain, retina and erythrocytes in breast - and formula-fed infants. Am $\mathcal{F}$ Clin Nutr 1994; 60: 189-94.

4 Fisher SK, Agranoff BW. Receptor activation and inositol lipid hydrolysis in neural tissues. $\mathcal{F}$ Neurochem 1987; 48: 999-1017.

5 Araki T, Kato H, Hara H, Kogure K. Postischemic binding of $\left[{ }^{3} \mathrm{H}\right]$ phorbol 12,13 dibutyrate and $\left[{ }^{3} \mathrm{H}\right]$ inositol $1,4,5-$ of $\left[{ }^{3} \mathrm{H}\right]$ phorbol 12,13 dibutyrate and $\left[{ }^{3} \mathrm{H}\right]$ inositol $1,4,5-$
trisphosphate in the gerbil brain: an autoradiographic trisphosphate in the gerbil brain: an

$6 \mathrm{Henzi}$ V, MacDermott AB. Characteristics and function of $\mathrm{Ca}^{2+}$ and inositol 1,4,5-trisphosphate-releasable stores of $\mathrm{Ca}^{2+}$ and inositol 1,4,5-trisphosphate-releasable

7 Cullis PR, De Kruijff B. Lipid polymorphism and the functional roles of lipids in biological membranes. Biochim Biophys Acta 1979; 559: 399-420.

8 Cook AM, Low E, Ishijimi M. Effect of phosphatidylserine decarboxylase on neural excitation. Nature: New Biology 1972; 239: 150-1.

9 Fenske DB, Jarrell HC, Guo Y, Hui SW. Effect of unsaturated phosphatidylethanolamine on the chain order profile of bilayers at the onset of the hexagonal phase transition. A ${ }^{2} \mathrm{H}$ NMR study. Biochemistry 1990; 29: transition.

10 Salem N Jr, Kim HY, Yergey JA. Docosahexaenoic acid: membrane function and metabolism. In: Simopoulos AP, Artemis $\mathrm{P}$, et al, eds. Health effects of polyunsaturated fatty acids in seafoods. London: Academic Press, 1986: 263-317.

11 Orlacchio A, Maffei C, Binaglia L, Porcellati G. The effect of membrane phospholipid acyl-chain composition on the activity of brain $\beta-\mathrm{N}$-acetyl-D-glucosaminidase. Biochem $\mathcal{F}$ 1981; 195: 383-8.

12 Tanaka $\mathrm{R}$. Comparison of lipid effects on $\mathrm{K}^{+}-\mathrm{Mg}^{2+}$ activated p-nitrophenyl phosphatase and $\mathrm{Na}^{+}-\mathrm{K}^{+}-\mathbf{M g}^{2+}$ activated adenosine triphosphatase of membrane. f Neurochem 1969; 16: 1301-7.

13 Besley GTN, Gatt S. Spectrophotometric and fluorimetric assays of galactocerebrosidase activity, their use in the diagnosis of Krabbe's disease. Clin Chim Acta 1981; 110: 19-26.

14 O'Brien JS, Okada S, Chen A, Fillerup DL. Tay-Sachs disease. Detection and heteroxygotes and homozygotes by
serum hexosaminidase assay. Clin Chim Acta 1970; 283: serum 20 .

15 Raghavan SS, Tjopol J, Kolodny EH. Leukocyte $\beta$-glucosidase in homozygotes and heterozygotes for Gaucher's disease. Am ₹ Hum Genet 1980; 32: 158-73.

16 Farquharson J, Jamieson EC, Muir J, Cockburn F, Logan RW. Direct gas chromatographic assay of urinary medium-chain fatty acylcarnitines by their thermal decomposition. Clin Chim Acta 1992; 205: 233-40.

17 Roe CR, Millington DS, Maltby DA, Wellman RB. Postmortem recognition of inherited metabolic disorders from specific acylcarnitines in tissue in cases of sudden infant death [Letter]. Lancet 1987; i: 512 .

18 Suzuki Y, Orii T, Mori M, Tatibana M, Hashimoto T. Deficient activities and proteins of peroxisomal $\beta$-oxidation enzymes in infants with Zellweger syndrome. Clin tion enzymes in infants with

19 Svennerholm L, Vanier M. The distribution of lipids in the human nervous system. III. Fatty acid composition of phosphoglycerides of human fetal and infant brain. Brain Res 1973; 50: 341-51.

20 Hayes KC, Pronczuk A, Wood RA, Guy DG. Modulation of infant formula fat profile alters the low-density lipoprotein/high-density lipoprotein ratio and plasma fatty acid distribution relative to those with breast feeding. f Pediatr 1992; 120: 109-16.

21 Koletzko B, Schmidt E, Bremer HJ, Haug M, Harzer G.
Effects on dietary long-chain polyunsaturated fatty acids on the essential fatty acid status of premature infants. Eur 7 Pediatr 1989; 148: 669-75.

22 Farquharson J, Cockburn F, Patrick WA, Jamieson EC, Logan RW. Effect of diet on infant subcutaneous tissue triglyceride fatty acids. Arch Dis Child 1993; 69: 589-93.

23 Stubbs CD, Smith AD. The modification of mammalian membrane polyunsaturated fatty acid composition in relation to membrane fluidity and function. Biochim Biophys Acta 1984; 779: 89-137.

24 Salem N. Specialization in membrane structure and metabolism with respect to polyunsaturated lipids. In:
Karnovsky $\mathrm{ML}$, Leaf $\mathrm{A}$, Bolis LC, eds. Biological membranes: aberations in membrane structure and function. New Yo:k: Alan R Liss, 1988: 319-33.

25 Stubbs CD, Smith AD. Essential fatty acids in membrane: physical properties and function. Biochem Soc Trans 1990; 18: 779-81.

26 Food and Agricultural Organisation and World Health Organisation. The role of dietary fats and oils in human nutrition. (Report of an expert consultation.) Rome: FAO, 1978.

27 Bourre JM, Pascal G, Durand G, Masson M, Dumont O, Piciotti $M$. Alterations in the fatty acid composition of rat brain cells (neurons, astrocytes and oligodendrocytes) and of subcellular fractions (myelin and synaptosomes) induced by a diet devoid of n-3 fatty acids. $\mathcal{F}$ Neurochem 1984; 43: 342-8.

28 Galli C, Trzeciak HI, Paoletti R. Effects of dietary fatty acids on the fatty acid composition of brain ethanolamine
phosphoglyceride: reciprocal replacement of $n-6$ and $n-3$ polyunsaturated fatty acids. Biochim Biophys Acta 1971; 248: 449-54.

29 Mohrhauer H, Holman RT. Alteration of the fatty acid composition of brain lipids by varying levels of dietary essential fatty acids. $\mathcal{F}$ Neurochem $1963 ; 10: 523-30$

30 Voss A, Reinhart M, Sankarappa S, Sprecher H. The metabolism of $7,10,13,16,19$-docosapentaenoic acid to $4,7,10,13,16,19$-docosahexaenoic acid in rat liver is independent of a $\Delta 4$-desaturase. F Biol Chem 1991; 266: 19995-20000.

31 Sinclair AJ. The incorporation of radioactive polyunsaturated fatty acids into the liver and brain of the developing rat. Lipids 1975; 10: 175-84.

32 Porcellati G, Arienti G, Pirotta M, Giorgini D. Baseexchange reactions for the synthesis of phospholipids in nervous tissue: the incorporation of serine and ethanolamine into the phospholipids of isolated brain microsomes. F Neurochem 1970; 18: 1395-417.

33 Forsythe ID, Clements JD. Presynaptic glutamate receptors depress excitatory monosynaptic transmission between mouse hippocampal neurones. I Physiol (Lond) 1990; 429: $1-16$.

34 Nawy S, Jahr CE. Suppression by glutamate of cGMPactivated conductance in retinal bipolar cells. Nature 1990; 346: 269-71.

35 Slaughter MM, Miller RF. 2-Amino-4-phosphonobutyric acid: a new pharmacological tool for retinal research. Science 1981; 211: 182-5.

36 Lucas A, Morley R, Cole TJ, et al. Early diet in preterm babies and developmental status at 18 months. Lancet 1990; i: 1477-81.

37 Lucas A, Morley R, Cole TJ, Lister G, Leeson-Payne C. Breast milk and subsequent intelligence quotient in children born preterm. Lancet 1992; 339: 261-4.

38 Uauy R, Birch E, Birch D, Perano P. Visual and brain function measurements in studies in $n-3$ fatty acid

39 Carlson SE, Werkman SH, Peeples JM, Wilson WM. Long chain fatty acids and early visual and cognitive development of preterm infants. Eur $\mathcal{f}$ Clin Nutr 1994; 48: S27-30.

40 Makrides $M$, Simmer A, Goggin M, Gibson RA. Erythrocyte docosahexaenoic acid correlates with the visual response of healthy, term infants. Pediatr Res 1993; 33: 425-7.

41 Birch E, Birch D, Hoffman D, Hale L, Everett M, Uauy R. Breast feeding and optimal visual development. $\mathcal{F}$ Pediatr Ophthalmol Strabismus 1993; 30: 33-8.

42 Standing Committee on Nutrition of the British Paediatric Association. Is breast feeding beneficial in the UK? Arch Dis Child 1994; 71: 376-80.

43 Horrobin DF, Manku MS, Hillman H, Glen AI, Glen M. Fatty acid levels in the brains of schizophrenics and Fatty acid levels in the brains of schizophrenics

44 Soderberg M, Ediund C, Kristensson K, Dallner G. Fatty acid composition of brain phospholipids in aging and in Alzheimer's disease. Lipids 1991; 26: 421-5.

45 Brooksbank BWL, Martinez M. Lipid abnormalities in the brain in adult Down's syndrome and Alzheimer's disease. Mol Chem Neuropathol 1989; 11: 157-85.

46 Pisacane A, Impagliazzo N, Russo $M$, et al. Breast feeding and multiple sclerosis. BMF 1994; 308: 1411-2. 